\title{
Two-Layer Model in Piezoelectric Photothermal Spectra of CdTe Crystals
}

\author{
J. Zakrzewski ${ }^{1}$ (1) $\cdot$ K. Strzałkowski ${ }^{1} \cdot$ M. Maliński ${ }^{2} \cdot$ Ł. Chrobak $^{2}$ \\ Received: 9 November 2017 / Accepted: 13 May 2019 / Published online: 24 May 2019 \\ (c) The Author(s) 2019
}

\begin{abstract}
Two-layer model was applied to interpret the piezoelectric photothermal spectra of bulk CdTe crystals. The assumption of the existence of the subsurface layer of the thermal parameters significantly different from the bulk part of the crystals explains the behavior of amplitude spectra in high absorption region. It was recognized that the thickness of this layer is about $0.5 \mu \mathrm{m}$. It is the result of the surface treatment of the sample: grinding, polishing and etching. The thermal and optical parameters of this layer are presented and discussed.
\end{abstract}

Keywords Piezoelectric detection · Photothermal spectroscopy $\cdot$ Semiconductors

\section{Introduction}

Wide gap II-VI semiconductors are still in the center of interests as they can be applied in bandgap engineering, interband and intersubband transitions, diluted materials and devices [1-3]. The mixing of the compounds during the procedure of growth gives the possibility to change electronic and thermal properties, lattice parameters and bandgap energies.

Cadmium telluride (CdTe) is one of the promising materials for high efficiency, low-cost thin-film solar cells. The mechanical and optical properties of single crystals depend on various factors such as the quality of the starting material, heat treatment process, pulling rate of ampoule and growth temperature.

Based on CdTe, mixed crystals are called "semimagnetic semiconductors" or "diluted magnetic semiconductors (DMS)." In this kind of materials, one component is a non-magnetic semiconductor and the second is magnetic one. Among beryllium

\footnotetext{
$凶 \quad$ J. Zakrzewski

jzakrzew@fizyka.umk.pl

1 Institute of Physics, Nicolaus Copernicus University, ul.Grudziądzka 5/7, 87-100 Toruń, Poland

2 Faculty of Electronics and Computer Science, Technical University of Koszalin, ul. Sniadeckich 2, 75-453 Koszalin, Poland
} 
chalcogenides, BeS has very high hardness and BeTe is a p-type semiconductor of the zinc blende structure.

Among others, also photothermal methods are applied to the investigations of semiconducting materials [4]. One of them is a piezoelectric technique (PZE). PZE is very sensitive and considered to be a direct method of measurements as the signal depends on the quantum yield of the non-radiative transitions in semiconductors-only the energy absorbed by the sample produces the photothermal signal. It is also a complementary technique to the absorption and photoluminescence spectroscopy $[4,5]$. This detection and the entire experiment are easy to perform thanks to the standard phase-sensitive (lock-in) technique used, but it is difficult to quantify the interpretation.

Recently, it has been observed that the preparation process strongly influences the photoacoustic spectra [6,7]. The aim of this article is to present and interpret the influence of the surface parameters on the PZE amplitude and phase spectra of CdTe samples.

Cadmium telluride and its mixed alloys have been already investigated by the photothermal methods, but there is still an area to improve the interpretation of the experimental data. Delgadillo et al. [8] investigated the correlation between the surface roughness and the surface recombination velocity. They used the microphone detection and the frequency-domain experimental data. Geode et al. $[9,10]$ used the piezo-detection to measure CdTe and $\mathrm{CdTe} / \mathrm{Hg}, \mathrm{ZnS} / \mathrm{Mn}, \mathrm{CdS} / \mathrm{Se}$ and $\mathrm{CdS} / \mathrm{Te}$. They did not propose any theoretical model and showed only amplitude data of their experiments. The authors did not give any information of the preparation of the surfaces that can be responsible for the observed changes of the amplitude. For all the materials, the decrease in the amplitude in the high absorption region (above the energy gap) is observed. Goede interpreted the maximum in this region as associated with the presence of free excitons and their deexcitation in the vicinity of the surface. The decrease in the signal in the high absorption region in the case of $\mathrm{CdS} / \mathrm{Se}$ was interpreted as the result of the increase in the disorder of the crystal structure with the increase of Se concentration.

The decrease in the amplitude of the photothermal signal above the energy gap was also observed by Matsumori et al. [11]. They investigated the influence of the damages caused by the implantation of ions in Si. Photothermal signal proved to be sensitive to the dimension and damage structure in the implanted layer. The authors associated these features with the change of the thermal diffusion length in the damaged layer.

The processes of the surface preparation were also investigated in the case of silicon: after mechanical treatment $[12,13]$ and polishing [14]. The crystal lattice in the subsurface layer has been damaged - the multiple phase transformations $[15,16]$, dislocations [17] and stacking faults [18] were observed. The damaged layer was also investigated in the case of silicon nitride [19] and silicon carbide [20].

The thermal parameters of a damaged layer are different than the rest part of the sample. Cabrero et al. [21] investigated the influence of a heavy ion irradiation of silicon carbide on the value of thermal conductivity. In the most defective part of the sample, the thermal conductivity was about one hundred times smaller than in the case of a pure silicon carbide. Terris et al. [22] studied the anisotropy of silicon nanostructures and observed the increase in the thermal conductivity values depending on the thickness of damaged layer. 
The changes of thermal conductivity depending on the composition and doping in semiconductors were investigated by Verma et al. [23] and Estreicher et al. [24]. Storm et al. [25] explored the procedures and quality of the preparation for (001) ZnSe surface for homoepitaxy: after the procedure of cutting, mechanical polishing, mechanic-chemical polishing and chemical polishing. The thickness of a damaged layer caused by polishing was estimated by the high-resolution Roentgen diffraction. Its value was computed as $10 \mu \mathrm{m}$. The removal of this layer by chemical etching leads to elimination of the surface defects.

The evaluation of damaged layer after polishing procedure for A2B6 semiconductors $\mathrm{ZnSe}, \mathrm{CdS}$ and $\mathrm{ZnO}$ was carried out by Lucca et al. [26-28]. The subsurface layer in the optical active materials was investigated by Shen et al. [29]. The influence of polishing and ultra-precise treatment of A3B5 GaAs, GaP and InP semiconductors was investigated by Venkatesh et al. [30].

\section{Sample Preparation}

Samples were grown from the high-purity powder with the high-pressure Bridgman method. The crystal rod was cut into about $1 \mathrm{~mm}$ thick samples which were first grinded using grinding powder (size in diameter $10 \mu \mathrm{m}$ ), then polished with a diamond paste $(1 \mu \mathrm{m})$ and finally chemically etched. Solution of $\mathrm{H}_{2} \mathrm{SO}_{4}(96 \%), \mathrm{K}_{2} \mathrm{Cr}_{2} \mathrm{O}_{7}$ and water was used for etching the samples. After etching, the samples were rinsed in distilled water and then put for a few seconds in boiling $\mathrm{NaOH}$. Then, the samples were rinsed again in cold and next in boiling distilled water and finally in ethyl alcohol.

\section{Theoretical Formulae Used for Computations}

Theoretical formulae elaborated and used for computations of the piezoelectric spectra of the two-layer structure are based on the earlier papers: The temperature spatial distribution formula used for computations of the piezoelectric spectra was published in paper [31]. Application of this formula, for the temperature distribution, for computations of piezoelectric spectra was presented in paper [32]. The temperature distribution formula for computations of the piezoelectric spectra of silicon samples was presented in paper [33]. Derivation of the formulae for the temperature distributions in two-layer system, e.g., a surface layer and the substrate, was presented in paper [34].

For computations of the piezoelectric spectra of a two-layer system, the formula (1), shown below, was used [34]. It is the development of the Jackson and Amer formula [35] for the generation of the piezoelectric signal of the two-layer system. It assumes that the signal is produced due to temperature distributions in the sample and the surface layer. 


$$
\begin{aligned}
\operatorname{PZE}\left(E_{p}\right)= & \frac{1}{L} \cdot\left(\int_{0}^{l} T_{s}\left(x, E_{p}\right) \cdot \partial x\right)-\frac{6}{L^{2}} \cdot \int_{0}^{l}\left(\frac{L}{2}-x\right) \cdot T_{s}\left(x, E_{p}\right) \cdot \partial x \\
& +\frac{1}{L} \cdot\left(\int_{l}^{L} T_{p}\left(y, E_{p}\right) \cdot \partial y\right)-\frac{6}{L^{2}} \cdot \int_{l}^{L}\left(\frac{L}{2}-y\right) \cdot T_{p}\left(y, E_{p}\right) \cdot \partial y
\end{aligned}
$$

where $T_{s}\left(x, E_{p}\right)=T_{s}\left(x, f, \alpha_{s}, \lambda_{s}, E_{p}, \beta_{s}\left(E_{p}\right), l\right)$ is the temperature spatial distribution in the surface layer, $T_{p}\left(y, E_{p}\right)=T_{p}\left(y, f, \alpha_{p}, \lambda_{p}, E_{p}, \beta_{p}\left(E_{p}\right), L\right)$ is the temperature spatial distribution in the substrate, $x$ is the spatial coordinate, $f$ is the frequency of modulation, $\alpha$ is the thermal diffusivity, $\lambda$ is the thermal conductivity, $E_{\mathrm{p}}$ is the energy of photons, $\beta$ is the optical absorption coefficient, $l$ is the thickness of the surface layer, and $L$ is the thickness of the whole structure, i.e., a surface layer and the substrate. Indexes $s$ and $p$ mean the surface layer and the substrate, respectively.

\section{Experimental Results and Their Numerical Interpretation}

The experimental setup used for investigations was typical for spectroscopic measurements based on a phase-sensitive (lock-in) detection. It was described in detail in [35]. The cell (with the PZT detector) used in experiments, allowing for the measurements in a front and a rear mode, was described in [7, 37]. The rear and front configurations in piezoelectric detection are associated with the geometry of sample and a detector position. At the rear mode, the sample is irradiated from one side and the detector is located on the other (non-illuminated); at front mode detector is located at the illuminated surface. As the state of surfaces of the sample can differ slightly due to the preparation process, the whole set of measurements can cover the four spectra: front and rear amplitude and phase spectra with the illumination of one and then the other side. The sides are freely chosen, but the spectra are assigned to the illumination of a given side and a configuration (front and rear).

Experimental and theoretical (one-layer model, [37]) piezoelectric amplitude and phase spectra of CdTe sample, at $f=40 \mathrm{~Hz}$ in the rear configuration, are presented in Fig. 1a, b, respectively. The frequency chosen gives the high and stable photothermal signal. The higher the frequency of modulation, the smaller the thermal diffusion length, and the signal is generated at the smaller thickness of the sample. $40 \mathrm{~Hz}$ frequency enables the generation of the signal in both layers of the sample.

One can see that the theoretical characteristics do not fit to the experimental data. In experimental amplitude, the decrease in the amplitude of the signal in a high absorption region is observed. The same feature was observed by Goede [10] and Matsumori [11]. The application of a two-layer model adjusts the experimental data to the theory.

Theoretical characteristics and their fit to the experimental characteristics computed in the two-layer model of the sample with a damaged surface are presented in Fig. 2a, b.

In a two-layer model, the surface layer and the substrate are described each by the following sets of thermal and optical parameters: thermal diffusivity, thermal conductivity, thickness of the surface layer and optical absorption coefficient spectrum. 
Fig. 1 Experimental and theoretical piezoelectric amplitude (a) and phase (b) spectra of CdTe sample at $f=$ $40 \mathrm{~Hz}$ in the rear configuration. Circles are experimental data and solid lines are theoretical characteristics in the model of the ideal sample
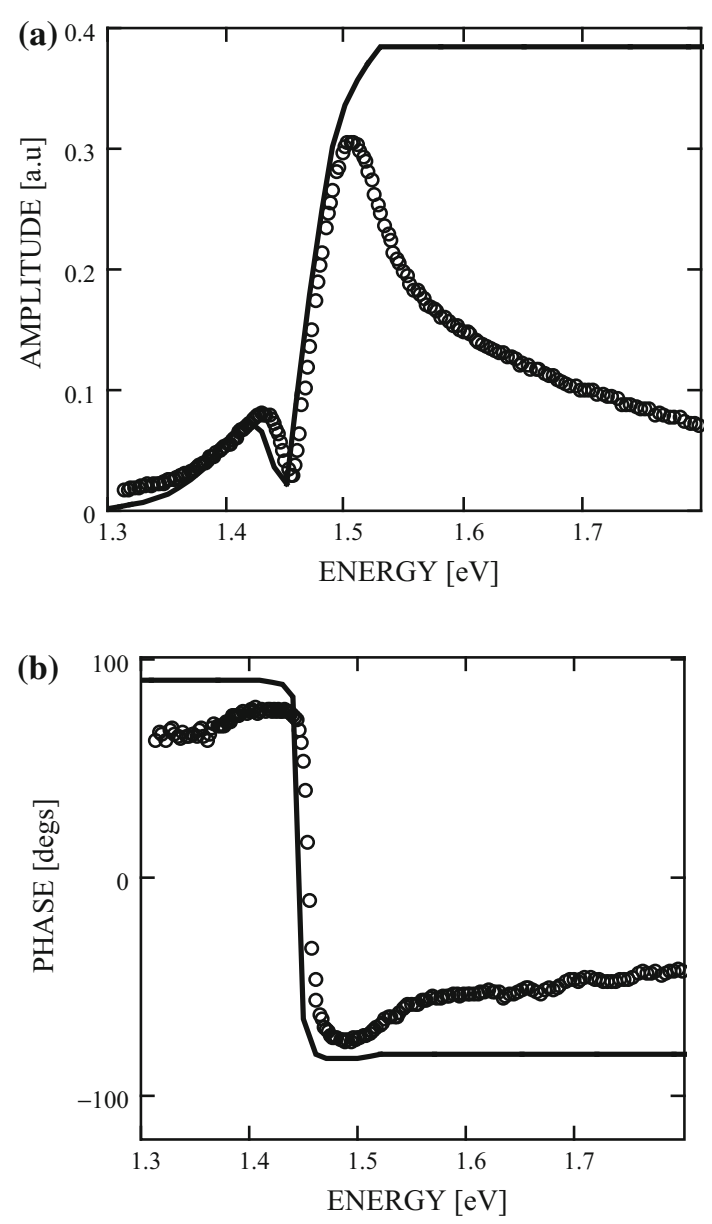

From the fitting of theoretical characteristics to the experimental characteristics, the following thermal and optical parameters of the surface layer and the substrate were determined:

\subsection{Thermal Parameters}

Thickness of the surface (damaged) layer $l=0.5 \mu \mathrm{m}$.

Thermal diffusivity and thermal conductivity of the surface layer $\alpha_{s}=0.001 \mathrm{~cm}^{2} \mathrm{~s}^{-1}, \lambda_{s}=0.012 \mathrm{cal} \mathrm{s}^{-1} \cdot \mathrm{cm} \cdot \mathrm{K}$

Thermal diffusivity and thermal conductivity of the substrate.

$\alpha_{p}=0.1 \mathrm{~cm}^{2} \mathrm{~s}^{-1}, \lambda_{p}=0.012 \mathrm{cal} \mathrm{s}{ }^{-1} \cdot \mathrm{cm} \cdot \mathrm{K}$

Thermal reflection coefficient between the surface layer and the substrate computed for the above thermal diffusivities and thermal conductivities $R_{12}=0.818$. 
Fig. 2 Experimental and theoretical piezoelectric amplitude (a) and phase (b) spectra of CdTe sample at $f=$ $40 \mathrm{~Hz}$ in the rear configuration. Circles are experimental data and solid lines are theoretical characteristics computed in the two-layer model of the sample with a damaged surface
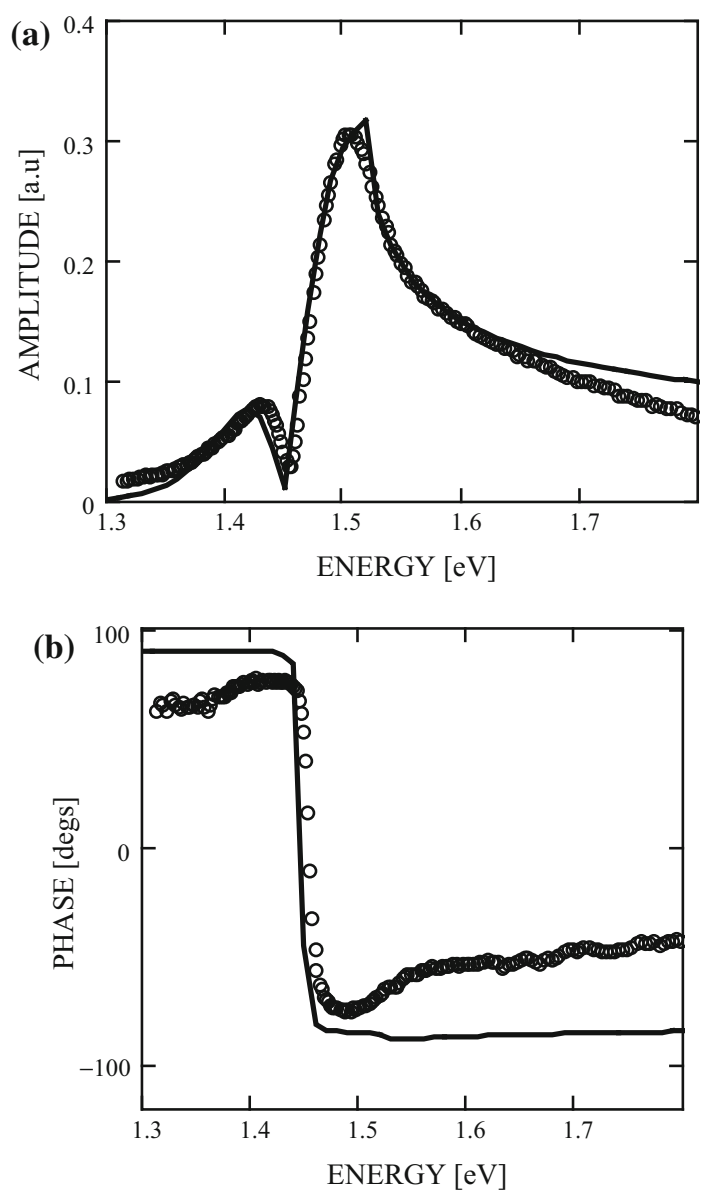

\subsection{Optical Parameters}

Optical absorption coefficient factor and the energy gap of the surface layer. $A_{s}=90000 \mathrm{~cm}^{-1} \cdot \mathrm{eV}^{-1 / 2}, E g_{s}=1.52 \mathrm{eV}$

Optical absorption coefficient factor and the energy gap of the substrate.

$A_{p}=20000 \mathrm{~cm}^{-1} \cdot \mathrm{eV}^{-1 / 2}, E g_{p}=1.52 \mathrm{eV}$

Optical parameters of the Urbach absorption edge: $\beta_{0}=100 \mathrm{~cm}^{-1}, \gamma=0.9$ both for the surface layer and the substrate.

The analysis of the piezoelectric spectra of the same sample but in the front experimental configuration when the other side of the sample is illuminated is presented in Figs. 3, 4 and 5.

One can see again that the theoretical characteristics do not fit to the experimental characteristics computed in the ideal sample model (Fig. 3). The fitting of the theoretical characteristics to the experimental data for two thicknesses of the surface layer is presented in Figs. 4 and 5. 
Fig. 3 Experimental and theoretical piezoelectric amplitude (a) and phase (b) spectra of CdTe sample at $f=$ $40 \mathrm{~Hz}$ in the front configuration. Circles are experimental data and solid lines are theoretical characteristics computed in the one-layer model of the ideal sample
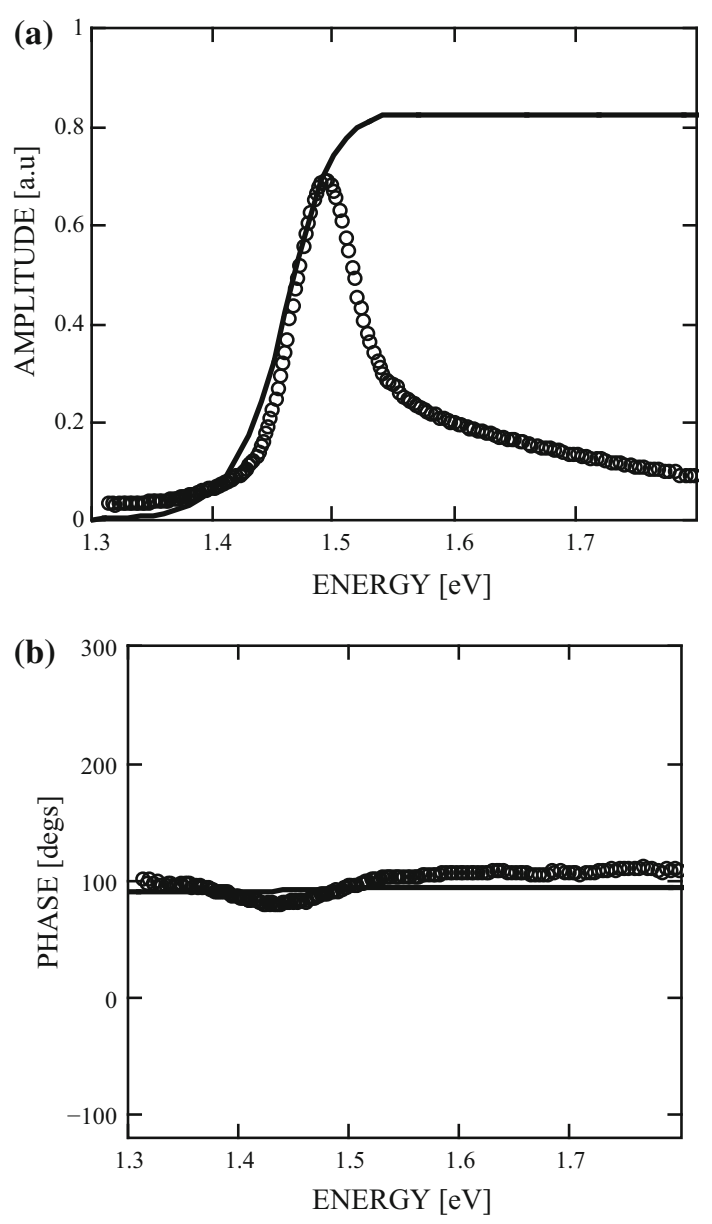

Theoretical characteristics were computed for the same set of parameters as for the rear configuration and the thickness of the surface layer $l=0.5 \mu \mathrm{m}$.

The fitting of theoretical characteristics to the experimental data for the same set of the thermal and optical parameters but for the thickness of the surface layer $l=0.7 \mu \mathrm{m}$ is presented in Fig. 5. The average standard deviation of the experimental amplitude spectra and the theoretical amplitude spectra obtained the values about $10 \%$ for the front configuration (Fig. 2a) and $20 \%$ for the rear configuration (Fig. 5a).

One can draw a conclusion that the thickness of the surface layer on the second side of the sample is larger than that on the first side.

\section{Conclusions}

Numerical analysis of the experimental piezoelectric spectra led us to the following conclusions. The thickness of the surface layer is about $0.5 \mu \mathrm{m}$. This is in the agree- 
Fig. 4 Experimental and theoretical piezoelectric amplitude (a) and phase (b) spectra of CdTe sample at $f=$ $40 \mathrm{~Hz}$ in the front configuration. Circles are experimental data and solid lines are theoretical characteristics computed in the two-layer model of the sample
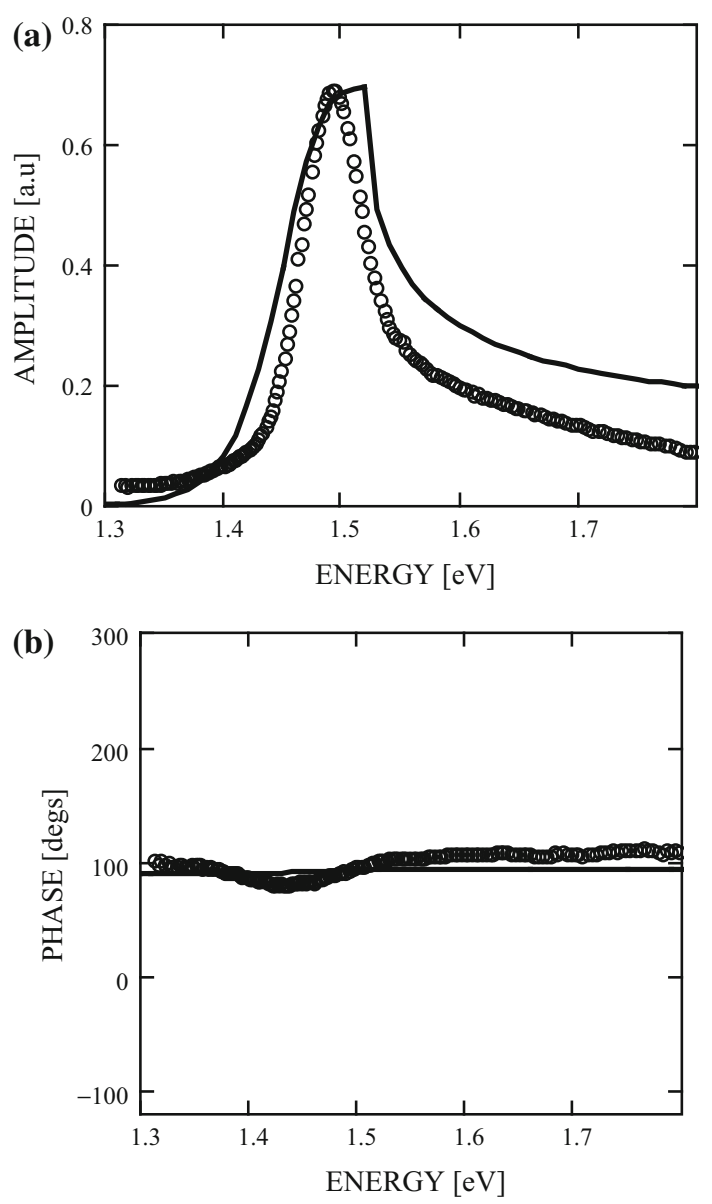

ment with the previous results obtained for measurements of the roughness by atomic force microscopy [36]. The thermal diffusivity of the surface layer is about 100 times smaller than the thermal diffusivity of the ideal crystal. That big change of the value of thermal diffusivity was previously observed for porous materials. In our case, it is caused by the mechanical and chemical procedure of the surface preparation and creation of the subsurface damaged layer. Thermal conductivity of both the surface layer and the ideal crystal is similar. Theoretical computations excluded the case that the thermal conductivity of the surface layer is 100 times smaller than the thermal conductivity of the ideal crystal as it could be expected. In such a case, an increase in the amplitude of the piezoelectric signal with the increase in the energy of photon would be observed for the theoretical characteristics instead of a decrease in the amplitude observed experimentally. Optical absorption of the surface layer is about four times larger than the optical absorption of the ideal CdTe crystal. To the best of our knowledge, these are the first theoretical piezoelectric spectra of a semiconductor sample computed in a two-layer model of the sample when the surface layer and the crystal are 
Fig. 5 Experimental and theoretical piezoelectric amplitude (a) and phase (b) spectra of CdTe sample at $f=$ $40 \mathrm{~Hz}$ in the front configuration. Circles are experimental data and solid lines are theoretical characteristics computed in the two-layer model of the sample
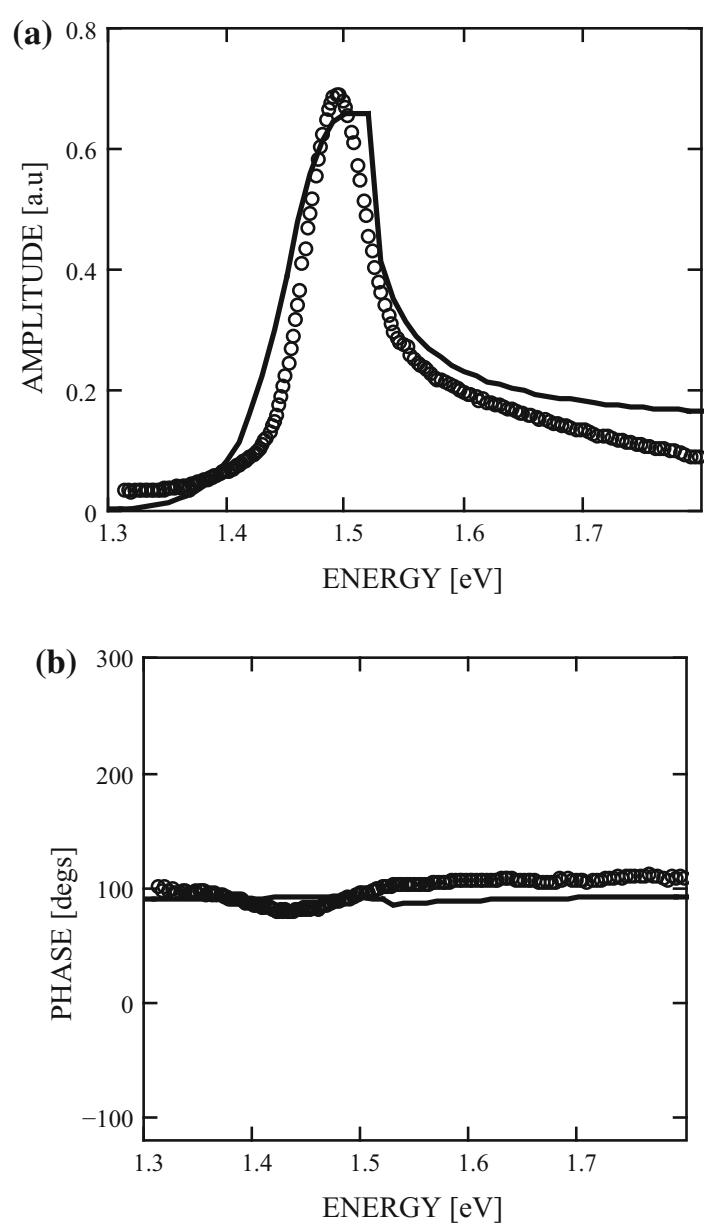

described by different sets of thermal and optical parameters. This approach enabled explanation of the observed decrease in the amplitude of the piezoelectric signal, for the photon energies above the energy gap of the crystal, observed experimentally for CdTe crystals.

The formula used in the interpretation uses only thermal effects of the photothermal signal generation. It is planned in the future to consider also the other effects associated with plasma waves and a surface recombination. The theoretical fittings show the good compliance with the experiments. The obtained values of parameters must be considered as the preliminary ones. Consideration of not included effects will allow to obtain the better fittings and more precise values of thermal parameters.

Open Access This article is distributed under the terms of the Creative Commons Attribution 4.0 International License (http://creativecommons.org/licenses/by/4.0/), which permits unrestricted use, distribution, and reproduction in any medium, provided you give appropriate credit to the original author(s) and the source, provide a link to the Creative Commons license, and indicate if changes were made. 


\section{References}

1. N. Zhang, A. Yeckel, A. Burger, Y. Cui, K.G. Lynn, J.J. Derby, J. Cryst. Growth 325, 10 (2011)

2. H. Choi, J. Korean Phys. Soc. 66, 27 (2015)

3. R. Rafiei, M.I. Reinhard, A. Sarbutt, S. Uxa, D. Boardman, G.C. Watt, E. Belas, K. Kim, A.E. Bolotnikov, R.B. James, J. Semicond. 34, 073001 (2013)

4. F.Z. Satour, A. Zegadi, J. Lumin. 132, 1688 (2012)

5. H. Benamrani, F.Z. Satour, A. Zegadi, A. Zouaoui, J. Lumin. 132, 305 (2012)

6. J. Zakrzewski, M. Maliński, K. Strzałkowski, Int. J. Thermophys. 33, 1228 (2012)

7. K. Strzałkowski, S. Kulesza, J. Zakrzewski, M. Maliński, Appl. Surf. Sci. 290, 27-34 (2014)

8. I. Delgadillo, M. Vargas, A. Cruz-Orea, J.J. Alvarado-Gil, R. Baquero, F. Sainchez-Sinencio, H. Vargas, Appl. Phys. B 64, 97 (1997)

9. O. Goede, W. Heimbrodt, Th Kopp, Phys. Status Sol. (a) 108, 443 (1988)

10. O. Goede, W. Heimbrodt, F. Sittel, Phys. Status Sol. (a) 93, 227 (1986)

11. T. Matsumori, M. Uchida, H. Yoshinaga, J. Kawai, T. Izumi, F. Uehara, Photoacoustic and Photothermal Phenomena III, vol. 69, Springer Series in Optical Sciences (Springer, Berlin, 1992), p. 357

12. J. Yan, T. Asami, T. Kuriyagawa, Procedia Eng. 32, 186 (2008)

13. A.Q. Biddut, L.C. Zhang, Y.M. Ali, Z. Liu, Scr. Mater. 59, 1178 (2008)

14. Z.J. Pei, Int. J. Mach. Manuf. 42, 238 (2002)

15. L.C. Zhang, I. Zarudi, Int. J. Mech. Sci. 43, 1985 (2001)

16. I. Zarudi, L.C. Zhang, Tribol. Int. 32, 701 (1999)

17. I. Zarudi, L.C. Zhang, J. Mater. Sci. Lett. 15, 586 (1996)

18. I. Zarudi, L.C. Zhang, J. Mater. Process. Tech. 84, 149 (1998)

19. W. Kanematsu, Wear 256, 100 (2004)

20. J.A. Patten, J. Jacob, J. Manuf. Process. 10, 28 (2008)

21. J. Cabrero, F. Audubert, R. Pailler, A. Kusiak, J.L. Battaglia, P. Weisbecker, J. Nucl. Mater. 396, 202 (2010)

22. D. Terris, K. Joulain, D. Lemonnier, D. Lacroix, P. Chantrenne, Int. J. Therm. Sci. 48, 1467 (2009)

23. A.S. Verma, B.K. Sarkar, S. Sharma, R. Bhandari, V.K. Jindal, Mater. Chem. Phys. 127, 74 (2011)

24. S.K. Estreicher, T.M. Gibbsons, Phys. B 404, 4509 (2009)

25. S. Storm, W. Neumann, H. Niehus, J. Crys, Growth 197, 517 (1999)

26. D.A. Lucca, R.L. Rhorer, C.J. Maggiore, Y.W. Seo, Ann. CIRP 44, 513 (1995)

27. D.A. Lucca, C.J. Maggiore, Ann. CIRP 46, 485 (1997)

28. D.A. Lucca, C.J. Wetteland, A. Misra, M.J. Klopfstein, M. Nastasi, C.J. Maggiore, J.R. Tesmer, Nucl. Instrum. Methods Phys. Res. B 219-220, 611 (2004)

29. J. Shen, S. Liu, K. Yi, H. He, J. Shao, Z. Fan, Optik 116, 288 (2005)

30. V.C. Venkatesh, I. Inasaki, H.K. Toenshof, T. Nakagawa, I.D. Marinescu, Ann. CIRP 44, 611 (1995)

31. M. Maliński, Arch. Acoust. 27, 217 (2002)

32. M. Maliński, Arch. Acoust. 28, 43 (2003)

33. M. Maliński, T. Ikari, Microelectron. J. 34, 1119 (2003)

34. M. Maliński, Eur. Phys. J. Spec. Top. 153, 291 (2008)

35. W. Jackson, N. Am. J. Appl. Phys. 51, 3343 (1980)

36. M. Pawlak, J. Gibkes, J.L. Fotsing, J. Zakrzewski, M. Maliński, B.K. Bein, J. Pelzl, J. de Physique IV 117, 47-56 (2004)

37. M. Maliński, J. Zakrzewski, K. Strzałkowski, Int. J. Thermophys. 28, 229 (2007)

Publisher's Note Springer Nature remains neutral with regard to jurisdictional claims in published maps and institutional affiliations. 